\title{
Special feature: perspectives on data science for advanced statistics
}

\author{
Makoto Aoshima ${ }^{1}$
}

Published online: 22 May 2018

(C) Japanese Federation of Statistical Science Associations 2018

Data science is certainly a hot term right now. Some $\mathrm{PhD}$ students mentioned that to get a job, you should list "Data Science" on your PhD whether you know anything about it or not! It seems to be becoming increasingly known that there are a lot of unqualified "data scientists" out there.

What is "Data Science"? I took the following snippet from the Wikipedia page on "Data Science": 'to extract knowledge or insights from data'. For me, it is really interesting that this is so similar to the definition of statistics that I offer, and have been offering for the past 30 years, when I teach elementary statistic courses.

That being said, is there any value added from this new terminology? I feel that there is, and the reason is that by renaming statistics it somehow seems to free up folks to start thinking about statistical matters, especially those who would probably not do so without that naming. In particular, many very talented people from fields such as math, computer science, and various areas of engineering want to call themselves "Data Scientists", and they are in the process of bringing lots of exciting new ideas, approaches, and ways of thinking to statistics.

The aim of this special feature is to gain perspectives on data science for advanced statistics. We have nine invited articles appearing in order of submission/acceptance.

In the article "Locally stationary spatio-temporal processes", Professors Yasumasa Matsuda and Yoshihiro Yajima propose a locally stationary spatiotemporal process to analyze the example of US precipitation data, which is a huge dataset composed of monthly observations of precipitation on thousands of monitoring points scattered irregularly all over the US.

In the article "Estimation pitfalls when the noise is not i.i.d.", Professors Liudas Giraitis, Masanobu Taniguchi, and Murad S. Taqqu extend Whittle estimation to

Makoto Aoshima

aoshima@math.tsukuba.ac.jp

http://www.math.tsukuba.ac.jp/ aoshima-lab/

1 Institute of Mathematics, University of Tsukuba, Tsukuba, Japan 
linear processes with a general stationary ergodic martingale difference noise. They raise awareness of the impact of a non-i.i.d. noise in applied work.

In the article "Stream-suitable optimization algorithms for some soft-margin support vector machine variants", Professors Hien D. Nguyen, Andrew T. Jones, and Geoffrey J. McLachlan discuss the stochastic majorization-minimization algorithm which allows for the training of SVMs on streamed data. They develop algorithms for training hinge loss, squared-hinge loss, and logistic loss SVMs.

In the article "A new era of statistics and data science education in Japanese universities", Professor Akimichi Takemura introduces the new faculty of data science at Shiga University, which launched an undergraduate program in 2017. This faculty emphasizes the study and application of statistics and is the first of its kind in Japan. He discusses the background of the developments and the prospects of statistics and data science in Japan.

In the article "Data science vs. statistics: two cultures?", Professors Iain Carmichael and J.S. Marron examine how different and evolving approaches to data analysis are related to broader trends in data science. They discuss how these trends relate to academic statistics, including future directions for communication, education, and research.

In the article "Divide and recombine (D\&R) data science projects for deep analysis of big data and high computational complexity", Professors Wen-wen Tung, Ashrith Barthur, Matthew C. Bowers, Yuying Song, John Gerth, and William S. Cleveland introduce the Divide and Recombine (D\&R) algorithm as a statistical approach to handle big, complex data. They discuss two big datasets, one from network cybersecurity and the other from climate science.

In the article "Partial quasi-likelihood analysis", Professor Nakahiro Yoshida generalizes the quasi-likelihood analysis to the partial quasi-likelihood analysis. Limit theorems for the quasi-likelihood estimators, especially the quasi-Bayesian estimator, are derived in the situation where the existence of a slow mixing component prohibits the Rosenthal-type inequality from applying to the derivation of the polynomial-type large deviation inequality for the statistical random field.

In the article "Multiplicity adjustment for temporal and spatial scan statistics using Markov property”, Professors Satoshi Kuriki, Kunihiko Takahashi, and Hisayuki Hara discuss computation of the conditional expectation in a multinomial distribution. The problem is motivated by the evaluation of the multiplicity-adjusted $p$ value of scan statistics in spatial epidemiology.

In the article "Sharing hash codes for multiple purposes", Professors Wiktor Pronobis, Danny Panknin, Johannes Kirschnick, Vignesh Srinivasan, Wojciech Samek, Volker Markl, Manohar Kaul, Klaus-Robert Müller, and Shinichi Nakajima propose multiple purpose LSH (locality sensitive hashing), which shares hash codes for different dissimilarities. They theoretically and empirically analyze the performance of multiple purpose LSH and demonstrate its usefulness on real-world data sets.

In the modern age, as the field of data science has grown, so has pressure on our own human resources and time. On the release of this first special feature, the journal team and publisher would sincerely like to thank the authors of the invited articles for very kindly taking the time to submit their research and support our fledgling journal. 